\title{
EL «ESTÁNDAR»: TIPOLOGÍA Y DEFINICIONES. SU VINCULACIÓN CON LA NORMA
}

\author{
Carla Amorós Negre
}

\section{RESUMEN}

El objetivo de este trabajo es contribuir a la delimitación del concepto de «estándar», que ha influido en gran medida en la configuración de los tratados gramaticales que acometen la descripción de las lenguas. Se analizarán pormenorizadamente las distintas propiedades y características que se le han atribuido en diversas y, muchas veces, contradictorias definiciones, que han proporcionado tanto diccionarios de lingüística y manuales de sociolingüística como monografías especializadas.

En general, parece existir un consenso generalizado en que la gestación de las «variedades/lenguas/dialectos» estándares es fruto de un proceso de cambio lingüístico deliberado, conocido como estandarización lingüística, que compete a la política y planificación lingüística. Sin embargo, la noción de «estándar» está, para algunos estudiosos, próxima a la de «lengua común», mientras que para otros se trata de una auténtica norma prescriptiva irrealizable e idealizada. Indudablemente, en las diferentes caracterizaciones del estándar se vislumbran, por un lado, una orientación más tradicionalista, que resalta su carácter democrático y conciliador y, por otro, una postura más crítica, revisionista que concibe el estándar como un mecanismo impositivo, responsable del conflicto social, la infravaloración y la estigmatización del resto de variedades y de sus respectivos hablantes. Así las cosas, se trata de dos aproximaciones no tan irreconciliables, como al principio puede parecer.

Asimismo, se tratará la evidente relación que el estándar guarda con el también polémico concepto de «norma lingüística». De hecho, el debate en torno a la cuestión de la mono o policentricidad de algunas lenguas trasluce muchas veces la compleja vinculación entre ambos conceptos.

Palabras clave: estándar absoluto, estándar relativo, norma descriptiva, norma prescriptiva, planificación lingüística, codificación, estandarización, koiné.

\section{ABSTRACT}

This paper aims to contribute to the delimitation of the term «standard» as it is used in linguistics. This has notably influenced grammatical treatises in their endeavour to describe different languages. It is our purpose to analyse in thorough detail those features and properties that have been commonly assigned to this controversial issue in several, and often, contradictory definitions provided by dictionaries of linguistics, handbooks of sociolinguistics or specialised monographs.

It has been traditionally contended that the emergence of standard «varieties/ languages/ dialects» is due to a deliberate linguistic change, referred to as language 
standardisation, that is primarily connected with language policy and language planning. Yet, the idea of a «standard» is, for some scholars, close to a «common language», whilst, for others, it represents a real prescriptive norm, idealised and unattainable. Undoubtedly, there are different approaches as far as the description of the «standard» is concerned. On the one hand, there exists a more traditional stance which emphasises its democratic and conciliatory bias. On the other, a more critical and revisionist perspective that looks at the «standard» in terms of its imposition, its responsibility for the social conflict, as well as the underestimation and stigmatization of other varieties and speakers that it entails. In this vein, these are not, however, as irreconcilable as they might seemingly appear.

In addition, this paper tackles the obvious relationship that the «standard» maintains with the not less contentious concept of «norm». Actually, the debate on the subject of the monocentrity or policentrity of some languages does some times show the complex interconnection between them.

Key Words: absolute standard, relative standard, descriptive norm, prescriptive norm, language planning, codification, standardisation, koiné.

\section{INTRODUCCIÓN}

La estandarización lingüística es, sin duda, una realidad difusa que, como ocurre con la mayoría de variables sociolingüísticas, no puede definirse según parámetros o reglas categoriales e invariables. Se trata de un proceso motivado por factores de naturaleza social, en el que intervienen condicionantes externos a la dinámica de la lengua misma, por lo que su estudio se aborda más adecuadamente desde la perspectiva de la sociolingüística aplicada y, concretamente, dentro del marco de la política y la planificación lingüísticas (PPL).

Es evidente que en una época en la que se defiende la riqueza, dignidad y legitimidad de todas las variedades lingüísticas, el interés por el tratamiento de cuestiones referentes a las «lenguas, dialectos, variedades» estándares se topa con la reticencia de muchos lingüistas, atendiendo al carácter prescriptivo que la estandarización lleva implícito.

Sin embargo, la reivindicación y el triunfo del pluralismo y la diversidad lingüísticas no puede hacer olvidar al lingüista que, por razones extralingüísticas, tal equipolencia no se refleja en la vida social de las lenguas y son los llamados «estándares», los que gozan de mayor reconocimiento y prestigio social, económico y/o cultural, a los ojos de los hablantes.

En este sentido, no parece aventurado afirmar que son, precisamente, esos factores de naturaleza extralingüística e ideológica que condicionan el proceso estandarizador, los que dificultan sobremanera la sistematización y caracterización de los idealizados estándares. Tal dificultad, no obstante, no exime a los profesionales de las lenguas del deber de tratar de establecer cuáles son sus rasgos definitorios y, con 
ello, evitar, «la intromisión de los advenedizos como caballos en una cacharrería» (Prieto de los Mozos 1999, p. 247).

Este es, justamente, el propósito hacia el que camina este trabajo, que pretende aportar un poco más de luz con el análisis de las propiedades y las definiciones más recurrentes para el concepto de estándar, cuya indeterminación, e incluso contradicción, justifican la necesidad de volver continuamente sobre él.

En el caso concreto del inglés estándar Speicher y Bielanski 2000, p. 152 exponen:

While the term Standard English is bandied around with great regularity, scholars rarely specify whether they are referring to the written or spoken form. They also fail to both define and specify the lexical, phonological, and grammatical features that comprise it.

Pero, ante todo,

All standard languages, regardless of genetic affiliation, might have enough in common to legitimize investigating them as a category unto themselves (Joseph 1987, p. 9).

\section{EL ESTÁNDAR: CONJUNCIÓN DE ARTIFICIALIDAD E HISTORICIDAD}

Una de las constantes en la definición de las variedades estándares es su carácter sociohistórico, resultado de un proceso de estandarización a lo largo de la evolución de algunas lenguas (Haugen 1968; Hudson 1980; Leith 1983; Zamora Salamanca 1985; Downes 1984; Milroy y Milroy 1985; Joseph 1987; Cameron 1995; Deumert y Vandenbussche 2004, Méndez García de Paredes 2007 etc.), que incluye las conocidas fases de selección, codificación, elaboración y aceptación. Así, Rojo p. 1986, 34-35 define el español estándar como «la [variedad] más representativa de la lengua histórica que «se ha ido haciendo en el seno de nuestra tradición idiomática» (Martín Zorraquino 2001). Por ello, si las normas particulares de lo que Joseph denomina synechdochic dialect devienen en modelo idiomático, «deben corresponderse con el modo de ser habitual de una lengua, esto es, con la variación» (Méndez García de Paredes 2007).

Indudablemente, la estandarización no es una característica intrínsecamente lingüística, ni tampoco un universal cultural, sino más bien un parámetro propio de la tradición occidental judeo-cristiana (y de las aculturadas a esta) que da cuenta del desarrollo de algunas lenguas. Sin embargo,

Since hierarchization characterizes all linguistic behaviour, synecdoche is characteristic of many non-Western cultures: there is at least a sense of who speaks the language best, whether it is the inhabitants of a specific locale in the region, the chief, the shaman. (Joseph 1987, p. 61) 
Así pues, si bien la codificación y cultivación, características de todo estándar, requieren de una planificación consciente y una intervención deliberada, lo que les ha valido la etiqueta de «artificial» o «no natural» (Voloshinov 1929; Hudson 1980; Bakhtin 1981; Romaine 1988; Grace 1990; Pascual y Prieto de los Mozos 1998; Moreno Cabrera 2008, etc.), la emergencia de una variedad en estándar puede no ser el fruto de una mediación directa, sino de un devenir circunstancial en la historia de una lengua (Demonte 2003). Por esta razón, dicha caracterización como «artificial» debe ser matizada.

Los estándares son inherentes al funcionamiento de la lengua. No son procesos de imposición por parte de unos agentes externos al ser histórico de una lengua, pues esta no es una realidad autónoma que pueda desligarse de la comunidad lingüística que la ha constituido como tal. (Méndez García de Paredes 2008)

Esta cita ilustra la necesidad de aludir a dos conceptos clave, los language standards, a los que se refería Méndez García de Paredes y, por otro lado, los standard languages, cuya interrelación demuestra que tanto la artificialidad como la naturalidad son propiedades presentes en el proceso de estandarización lingüística, y que ambas posiciones no están tan alejadas entre sí como a primera vista pudiera parecer.

Efectivamente, en todas las sociedades se generan modelos idiomáticos, un componente universal no artificial, los llamados intrinsic standards (Haas 1982) o language standards (Joseph 1987), que Joseph 1991, p. 13 define de la siguiente manera:

Language standards are the normative judgements made about a particular language by whoever be the arbiters within a particular, and enforced by persons such as teachers, editors, and grammarians

Estos no son el fruto de un proceso deliberadamente planificado, sino que surgen por el mero ejercicio de la conciencia lingüística. Se trata, simple y llanamente, de la percepción y las valoraciones que los hablantes hacen de su lengua. Así pues, no es un comportamiento exclusivo de la civilización occidental, sino que aflora en todas las comunidades lingüísticas, cuando se percibe la heterogeneidad dialectal. Así se expresa Deborah Cameron 1995, p. xi:

Value judgements on language form part of every competent speaker's linguistic repertoire. One of the things that people know how to do with words is to evaluate them, and I can see no principled justification for neglecting or denying this metalinguistic ability [...] The beliefs about language that informs people use of it arguably fall within the scope of what descriptive linguists ought to be able to give an account of.

Sin embargo, los mencionados intrinsic o language standards son la base para la elaboración de un concepto particular, este sí propio de una 
determinada tradición cultural, la occidental y, por tanto, no universal: los superposed standards (Haas 1982) o standard languages (Joseph 1987), en cuya emergencia se ha intervenido mediante los procesos artificiales, que son muchas actividades que competen a la planificación de corpus y a la planificación de estatus. Por ejemplo, según constata Lara 1976, p. 21:

La codificación explícita de una norma depende de factores sociales y culturales que no siempre aparecen en las comunidades lingüísticas.

Así pues,

A standard language [a language for which a significant body of language standards have been produced] represents one particular, non-universal set of choices from the larger, universal set of possibilities. (Joseph 1991, p. 14)

Estas variedades estándares ${ }^{1}$ codificadas requieren de la enseñanza explícita, puesto que no se corresponden exactamente con la lengua materna de ningún hablante (Tauli 1968; Hudson 1980; Joseph 1987, 1991; Romaine 1988, Cardona 1988; Pascual y Prieto de los Mozos 1998; Trudgill 1999; Fairclough 2001, Agha 2003, etc.). A su vez, no es de extrañar que exista un acuerdo generalizado en que todo estándar descansa en la modalidad escrita ${ }^{2}$ (Cooper 1989; Gallardo 1978; Milroy y Milroy 1985; Joseph 1987; Penny 2000; Pascual y Prieto de los Mozos 1998, Moreno Cabrera 2008, etc.), si bien este hecho «no niega, ni mucho menos, que haya una forma estándar hablada» (Gallardo 1978, p. 97), una apreciación que comparten Bartsch 1985, 1987; Joseph 1987; Van Maarle 1997 y Trudgill 1999, entre otros.

Precisamente, el factor sociopolítico, religioso, económico y/o cultural, que subyace al proceso estandarizador, explica que se hable de una «ideología de la estandarización» (Milroy y Milroy 1985) muy potenciada por la educación. No en balde, como pone de relieve Joseph 1987, p. 82

Standards are not native, unconsciously acquired dialects, but secondarily and consciously learned.

1 Una gran parte de los especialistas en la materia emplea muchas veces «estándar» como modificador del sustantivo lengua (Tauli 1968; Lewandowski 1982; Alvar 1990; Alcaraz Varó y Martínez Linares 1997; Bernárdez 1999; Mújica 2004; etc.), mientras que otros se inclinan por el término variedad (Bartsch 1985, 1987; Cerdà 1986; Pascual y Prieto de los Mozos 1998, etc.) que es, a nuestro entender, la opción más adecuada puesto que considera al estándar una variedad más del complejo dialectal, si bien especial, «que goza, lo reconocemos, de mayor y más amplia aceptación social, pero que desde una perspectiva estrictamente lingüística no es superior a ningún otro dialecto, sino sólo diferente» (Silva Corvalán 2001, p. 31). Asimismo, es también muy frecuente la alternancia entre los sustantivos lengua y variedad (Zamora Salamanca 1985a; Joseph 1987, 1991; Crystal 1995; Moreno Fernández 1998; Martín Zorraquino 2001; Alba 2001; Demonte 2003, etc.).

2 «An unwritten system [...] would be again a methaphorical standard» (Joseph 1987, p. 6). 
La búsqueda de la uniformidad estructural y su dependencia del plano escrito de la lengua, unidas a la mencionada necesidad de una instrucción formal para el dominio de la variedad estándar, han llevado, lógicamente, a muchos especialistas a atribuirle el calificativo de «artificial» ${ }^{3}$, fruto de un proceso de ingeniería lingüística (Leich 1983). Así, Kratochvíl 1968, pp. 135-136, por ejemplo, afirma:

Modern Standard Chinese, as well as any other modern standard language, is necessarily a partly artificial system.

Esta opinión la sustenta también Haugen 1968, p. 268:

Standard languages are artefacts that result either from pruning or grafting the tree $[\ldots]$.

En este sentido, cabe precisar que tal adjetivo no poseía, en sus comienzos, una connotación peyorativa, aunque es cierto que no ostentaba la virtud y nobleza que caracterizaba a los dialectos. «Artificial» remitía a un producto de la historia humana. Significaba, simplemente, que una lengua había sido reducida a arte, propósito que persigue Dante con su De vulgari eloquentia o Nebrija con la redacción de su Gramática de la lengua castellana (Joseph 1987).

Sucede, además, que en muchos trabajos lingüísticos se asiste a una desafortunada oposición entre «natural» (espontáneo, no planificado conscientemente) y «cultural» (planificado y con presencia de conciencia lingüística). Esta dicotomía, además de difícil de delimitar, corre el peligro de «rechazar fuera de la cultura, a la naturaleza, todo lo que no se conforma con la norma bajo la que se vive» (Lévi-Strauss 1952, p. 42). Tal y como advierte Joseph 1987, p. 11:

The two are inseparable. Nature is constituted by the entire universe, and culture is constituted by human consciousness, which is certainly part of the universe $[\ldots]$. The fallacy is heightened by any general belief that either nature or culture is intrinsically and unconditionally better than the other.

A este respecto, tómese en consideración la opinión de Jespersen 1925, que reitera lo dicho ya por Schuchardt en el siglo XIX, esto es, que la artificialidad y la naturalidad de las lenguas es una cuestión de grado, $y$, en todas ellas, existen elementos arbitrariamente creados.

El deseo por establecer una férrea oposición entre natural y artificial, con las correspondientes cualidades positivas y negativas asociadas, respectivamente, es similar a la relación dicotómica que algunos estudiosos intentan establecer entre descripción y prescripción (Cameron

\footnotetext{
${ }^{3}$ «[...]All languages show such variation as to make any designation of a single standard in any language as being artificial», comenta Myhill 2004, p. 35.
} 
1995), sin percatarse de que se trata, más bien de un continuum presente desde las primeras gramáticas de la antigüedad clásica, (Haas 1982; Crystal 1995; Davies 1997, Prieto de los Mozos 1999, etc.).

Volviendo a la cuestión de la artificialidad de las lenguas estándares, su surgimiento es, muchas veces, consecuencia de una engineered emergence (Joseph 1987) o planned choice (Haji Omar 1991), como es el caso del hebreo moderno, la lengua indonesia o el finés. Por lo que respecta a este último:

[Standard Finnish] was fabricated by picking and choosing from several local dialects. Today's Standard Finnish was deliberately codified first from southwestern dialects, with elements of eastern dialects interwoven in the 1800 s with the popularity of the publication of the oral epic Kalevala in an easter Finnish variety (McWorther 2001, p. 65).

Haugen 1966 ilustra también el caso del nynorsk o landsmal ${ }^{4}$, uno de los dos estándares noruegos, cuyo principal artífice, el poeta y lingüista Ivar Aasen, configuró a partir de distintos dialectos hablados en zonas rurales y urbanas. Fue una variedad deliberadamente construida para alejarse de la influencia danesa que caracterizaba al bokmål o riksmal, más empleado en la literatura y propio de la clase media-alta.

No obstante, la emergencia de un estándar puede no ser resultado de una intervención deliberada en el devenir de una determinada variedad, sino de una determinada situación sociohistórica en una comunidad, que lo ha erigido como tal. Así sucedió con los estándares chino o japonés (Joseph 1987), ejemplos de un incidental choice (Haji Omar 1991) o circumstantial emergence (Joseph 1987).

Según constata Subačius 2004, p. 357:

We are accustomed to think that early dialect selection languages (those that selected their norm-dialect in Renaissance or slightly later) were developing more or less circumstantially, with no clearly evident interference by language planners. Late dialect selection languages (mostly those of nineteenth century-the Romanticism period) are known as being much more influenced by the conscious efforts of cultural activists, writers, linguists, and other language planners.

Pero, la dificultad que supone el deslinde entre «planificado» $\mathrm{e}$ «incidental» se constata en la propia interrelación de ambos tipos en la mayoría de casos documentados. De hecho,

If we examine the histories of Standard French and Standard Spanish, we find that both arose more or less circumstantially, but that before the emergence was securely fixed a questione della lingua generally open. [Since...]

\footnotetext{
${ }^{4}$ Myhill 2004, p. 34 se refiere a éste de la siguiente manera: «Nynorsk, an artificial amalgamation of various rural dialects developed by Ivar Aasen».
} 
the projection of hierarchical evaluations on to dialects which constitutes synecdoche $[\ldots]$ has strong roots in general behaviour (Joseph 1987, p. 61).

Por el contrario, si bien el holandés estándar apareció sin una mediación directa, parece ser que muy pronto, a juicio de Van Maarle 1997, p. 22:

Standard Dutch was artificially provided with, among other things, a case system, a system of adjectival declension [...], a conjunctive, and an imperative plural, $[\ldots]$ generally considered to be inspired by Latin and German.

En conclusión, si se reconoce, como propone Joseph 1987, que la estandarización lingüística es un proceso cultural, vinculado a un particular complejo de ideas, costumbres, creencias y actitudes ante la lengua, un concepto propio de la tradición occidental judeo-cristiana ${ }^{5}$, al que se han aculturado otras comunidades etnolingüísticas, y se hace hincapié en que «civilizations can grow in the absence of this tradition», en consecuencia:

By historicing the process which in the structuralist tradition was treated as ahistorical, we can interpret degrees of standardization as they ought to be interpreted, as having nothing to do with cultural «advancement» in any sort of general and objective sense, but merely with degree of historical progress along the path of acculturation. (Joseph 1991, p. 14)

De esta manera, tampoco es de extrañar que muchas definiciones enfaticen que las variedades estándares son la manifestación de ese ser histórico de la lengua, porque actúan como marco de referencia, parámetro en torno al cual se mide la variación. Se trata de la función referencial del estándar, bajo el que se unifican diferentes comunidades lingüísticas.

A nuestro entender, el carácter sociohistórico del proceso estandarizador, que representa una opción determinada por la civilización occidental, con el fin de dar cuenta del desarrollo y evolución sociohistórica de algunas lenguas, puede reconciliarse con el componente artificial que, indudablemente, lo configura como tal.

\section{El ESTÁNDAR: ¿DIALECTO O COMPLEJO DIALECTAL?}

Otra de las propiedades esgrimidas con mucha frecuencia en las reflexiones sobre la naturaleza del estándar es su carácter aglutinador, que acoge al resto de variedades. Así, para Lewandowski 1982, p. 201,

\footnotetext{
${ }^{5}$ Según Grace 1990, p. 8: «Joseph standard language is approximately the same thing as $[\ldots]$ a monoculture-dedicated language».
} 
La lengua estándar está por encima de la(s) lengua(s) coloquiales y los dialectos, [...es] el medio más amplio y extendido [...]; frente a los dialectos y sociolectos, [es] el medio de comunicación más abstracto y de mayor extensión social

En este mismo sentido argumenta Gallardo 1978, p. 94, cuando califica al idioma estándar de «bien público», más accesible a la comunidad lingüística que los dialectos, porque en ella «confluyen todas las versiones el idioma», una opinión que también comparte Hernández Alonso 2001:

La lengua estándar a todos los abarca [...] Es un conjunto de sistemas diversos utilizados por grupos (geográficos, culturales, sociales, generacionales y de sexo) diversos integrados entre sí.

De hecho, en muchas ocasiones, la distinción entre «dialecto»y «lengua estándar» se ha basado en la consideración de que los primeros no han sido objeto de codificación y, justamente, el carácter de lengua escrita del estándar le ha posibilitado ser el vehículo apropiado para diferentes funciones y trascender los límites dialectales.

Sin embargo, su carácter suprarregional (Alba 2001; Demonte 2003), en tanto que no se corresponde con el habla de un territorio en particular, se explica más que por englobar a todos los dialectos, por ser él mismo un dialecto «convencionalmente superpuesto al conjunto de variedades geográficas sociales y estilísticas de una lengua»(O'Grady, Dobrovolsky y Aronoff 1997), atendiendo a razones extralingüísticas, que dan cuenta del prestigio que lleva asociado. Además, en palabras de Garrido Medina 1996, p. 72:

Comprobamos que lo que se suele llamar lengua es en realidad un dialecto, otro dialecto que por hegemonía política más o menos aceptada culturalmente se presenta como único correcto, y se le llama lengua. [...] Por su origen es un dialecto; por su situación es un sociolecto. Es la variedad del grupo social que gobierna y escribe ${ }^{6}$.

Así, no sorprende que, en los últimos tiempos, muchos sociolingüistas, sobre todo del ámbito anglosajón, se hayan referido al inglés estándar como un dialecto o variedad dentro del conjunto de la lengua inglesa y, en concreto, como un dialecto social (Dittmar y otros 1988, Trudgill 1999, etc.), el propio de «los cultos» (Steger 1998) o, en palabras de Sledd 1969, «the English used by the powerful», que se mantie-

${ }^{6}$ Según comenta Bartsch 1985, p. 39 (la cursiva es mía), «with the creation of a standard, the other varieties become part of a new opposition, namely the opposition standard versus nonstandard, and acquire the negative position within this opposition; this means, they become dialects of the language», lo cual explica la etiqueta de «lengua» que se le aplica en muchas definiciones. 
ne como parte del «ideological state apparatus» (Downes 1984). Por ello, dado que la lingüística científica ha defendido a ultranza la igualdad potencial de todas las variedades y ha puesto de relieve que las diferencias entre el estándar y el resto de dialectos no son intrínsecamente lingüísticas, la visión de Hudson 1980, según la cual antes de la lengua estándar sólo había dialectos, es decir, variedades no estándares, no parece la más acertada.

Standard English is often referred to as the standard language. It is clear, however, that Standard English is not a language [...] is less than a language, since it is only one variety of English among many. [...] unlike other dialects, Standard English is a purely social dialect. Because of its unusual history and its extreme sociological importance, it is no longer a geographical dialect, even if we can tell that its origins were originally in the southeast of England. (Trudgill 1999, p. 118)

También Alba 2001 se refiere al español estándar como «un dialecto particular, que por lo general es el sociolecto alto de una zona geográfica determinada, en un estilo específico, el de mayor formalidad». Así las cosas, diferentes voces se han alzado también en contra de considerarlo propiedad de una minoría privilegiada y prefieren describirlo como «a great social bank on which we all draw and to which we all contribute» (Kingman 1988, p. 14); citado en Crowley 1989, p. 255. Si bien se acepta que «its use is a marker of social group membership» (Cox 1991, p. 31), Gallardo 1968, p. 95 opina que el no tener acceso al estándar es «un problema que afecta a los individuos hablantes más que al idioma mismo», y así no cree legítimo hablar de «dialecto de clase». Naturalmente, pero la lengua no puede desligarse de sus hablantes, y por esta misma razón, se podría desestimar que «son precisamente los dialectos subestándares, de poca accesibilidad, los que tienden a perpetuar la estratificación social» (Gallardo 1978, p. 95).

La asociación del estándar con la variante culta de la lengua se explica, muy probablemente, por su identificación, en los primeros tiempos, con el literary language (Crowley 1989). No en balde, los lingüistas del Círculo de Praga, pioneros en la formulación de la estandarología, basaron su estudio en la lengua literaria checa. Actualmente, pese a que este símil está mayoritariamente desestimado, las valiosas contribuciones de Garvin, Mathesius o Havránek en el establecimiento de las propiedades, actitudes y funciones que caracterizan al estándar son referencia obligada en este campo de investigación y no deben, en modo alguno, obviarse.

En este estado de cosas, la alusión a la variedad estándar como la propia de la clase social elevada, que ostentaba el mayor prestigio en la esfera de la vida pública, pronto motivó la aparición de referencia y equivalencias entre «estándar», «el buen lenguaje» o well-bred language (Wyld 1934). 
El propio Wyld, que se presentaba a sí mismo como un observador descriptivo y objetivo, en busca del rigor científico (Crowley 1989), llega a afirmar:

$\mathrm{RSE}^{7}$ [Received Standard English] is superior from the character of its vowel sounds, to any other form of English, in beauty and clarity.

Por descontado, desde la perspectiva que aquí se adopta, esto es, la sociolingüística, «todas las lenguas conocidas son sistemas simbólicos de igual valor nativo» (Appel y Muysken 1987, p.76), por lo cual se rechaza por imperativo teórico esa evaluación lingüística, atendiendo a factores de naturaleza intrínsecamente lingüística. Tal postura descuida el valor simbólico de las lenguas en el seno de la sociedad y no puede, sino contribuir a la estigmatización discriminación y desigualdad lingüístico-sociales. Sin embargo, opiniones como las citadas, muestran cómo pronto se produjo esa reduccionista identificación entre lengua estándar y lengua correcta, aspecto que se trata en el siguiente apartado.

De este modo, si bien son muy pocos los especialistas que sostienen esta visión instrumentalista, aún hay quienes continúan muy imbuidos de una doctrina prescriptiva muy conservadora y rígida, que se evidencia en afirmaciones como la siguiente de Honey 1997, p. 5:

Standard English is not merely one variety among many, but instead is a specially important and valuable variety which derives its value from a set of qualities which are not shared by other, non-standard dialects

Tampoco resultan muy apropiadas las definiciones que lo equiparan al cultured (OED 1933) o educated English (Quirk y otros 1985) atendiendo a la subjetividad, imprecisión y volubilidad del parámetro «nivel educativo» Crowley 1989. Sin embargo, la mayoría sí parece coincidir en que el estándar ha trascendido los límites de un dialecto geográfico, y se acepta que «may be spoken with an unrestricted choice of accent» (Strevens 1983, p. 88; Kingman 1988; Carter 1999). A este respecto, Trudgill 1999 matiza que el estándar inglés no tiene que ver con la pronunciación, sino más bien con ciertas idiosincrasias gramaticales. Así pues, no está confinado al acento más prestigioso, esto es, la Received Pronunciation, ni posee un vocabulario o estilo específico. Tampoco es un registro, sino que él mismo puede emplearse en distintos registros y admite cierta variación regional, que permite hablar de un Standard British English y de un Standard American English, por ejemplo.

${ }^{7}$ Fue en 1934, en The best English: A claim for the superiority of Received Standard English, cuando Wyld modificó el término Standard English por Received Standard English para referirse al «inglés de las clases más instruidas», junto al que se encontraba, en una escala inferior, el Modified Standard English, no tan exquisito, a su juicio, pero, al menos, «not Provincial or Regional [...].» (Wyld 1934, p. 606). 
Así las cosas, también existen voces discrepantes, como Speicher y Bielanski 2000, p. 148, quienes afirman:

In Great Britain, the standard is called Received Pronunciation or The Queen's English, and in the United States, the standard is called Standard American English or National Network English.

La cuestión acerca de la existencia de una variante oral estándar ${ }^{8}$ ha sido muy polémica y cuestionada, atendiendo a la uniformidad e invariabilidad a la que tiende un estándar, «more a construct, or something postulated, than something real» (Bartsch 1985, p. 32). Dado que no se materializa exactamente en el habla de nadie y posee una naturaleza más bien mítica y mitificada, varios lingüistas defienden la imposibilidad de hablar el estándar (Milroy y Milroy 1985; Grace 1990; Moreno Cabrera 2008).

Así las cosas, si bien estos autores tienen buena parte de razón, descuidan una de las propiedades definitorias que establecieron los lingüistas praguenses, esto es, la estabilidad flexible. Es esta elasticidad del estándar la que permite, como apunta Orlando Alba 2001, que en el español estándar aparezcan «una serie de elementos de carácter fonético, sintáctico y léxico», de carácter nacional, como el leísmo o el voseo que, en modo alguno, ponen en peligro la unidad fundamental que se sustenta en el componente universal, panhispánico.

En consecuencia, muchos lingüistas que apuestan por la flexibilidad del estándar, se refieren a él como un continuum (Bartsch 1985, 1987; Joseph 1987; Carter 1999; Trudgill 1999; Carbonero 2003a, 2003b; Österreicher 2004, 2006; Méndez García de Paredes 2007, 2008, etc.), cuyo grado más idealizado es, precisamente, the written standard, «nobody's native language [...which] gradually came to be adopted as a norm for speaking [...] particularly among the well-educated urban elite» (Van Maarle 1997, p. 15). Con ello, este estándar hablado estaría más o menos próximo al modelo escrito y sería más o menos formal, en función de la situación comunicativa. Paralelamente, según señala Van Marle 1997, p. 15, «as soon as the written standard developed a spoken variety, however, the dialects came into its influence», con lo que se produciría una retroalimentación, una influencia recíproca, tal y como se desprende del siguiente esquema, lo cual ha llevado a lingüistas como Bartsch 1937, p. 31 a hablar de la existencia de variedades «clearly $[. .$.$] accepted as standard, or still standard language, and some$ varieties [como...] approximate standard»:

\footnotetext{
${ }^{8}$ Según Crowley 1989, p. 115 fue Henry Sweet, quien definió el standard spoken English como «the theoretically received pronunciation of literary English».
} 


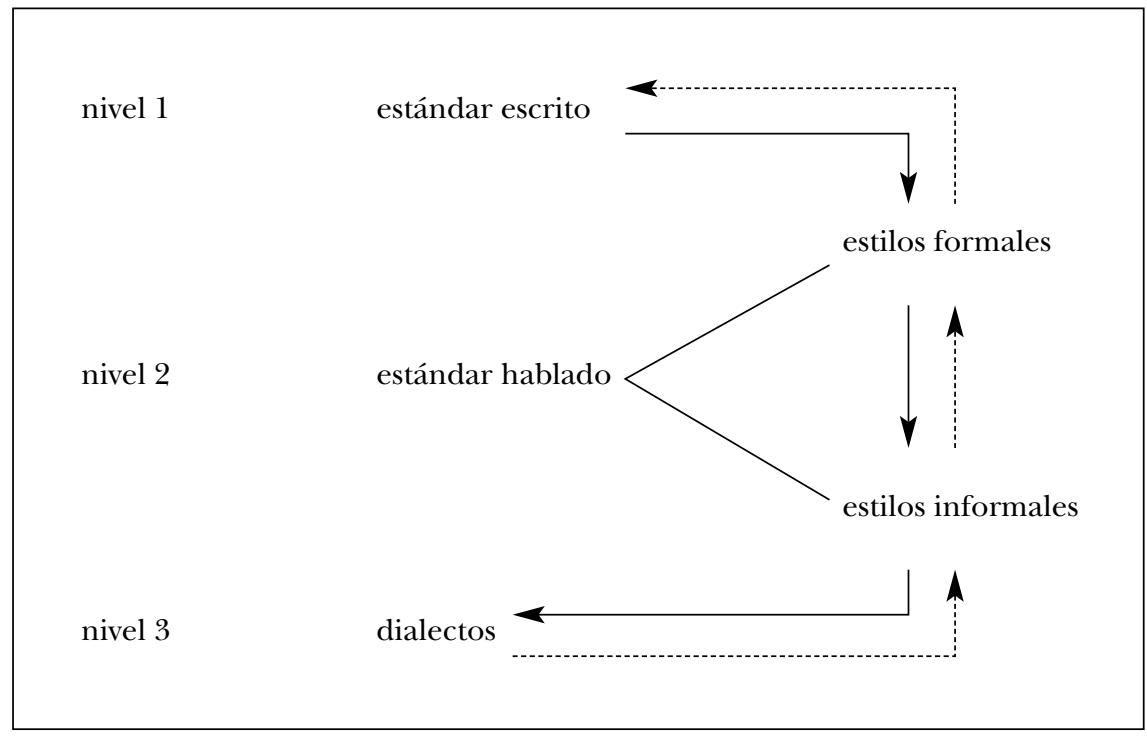

FIGURA 1: Relación entre las diferentes variedades lingüísticas. Fuente: Van Marle 1997, p. 19. [Mi traducción].

Así las cosas, no puede perderse de vista que el uso oral de la lengua se diferencia enormemente de los patrones que rigen el código escrito, un aspecto en el que insiste Chesire 1999, quien resalta la falta de estudios sobre la estructura del estándar oral y del lenguaje oral, en general.

Por consiguiente, disentimos de la opinión de Fernández Ramírez 1985, pp. 307-308 en el Prólogo a su Gramática Española [la cursiva es mía]:

[...] mi objetivo es el español común, el español cuidado que hablan las gentes cultas y universitarias de Madrid. Y entre ese español hablado y literario no existe, sobre todo en nuestros días, una distancia considerable. Las diferencias son más de léxico que de gramática.

En otro orden de cosas, es preciso también aludir a la tendencia que se tiene en juzgar las realizaciones orales con los patrones de un estándar codificado escrito, cuya planificación, contextualización y apoyo visual nada tienen que ver con los del medio oral (Milroy y Milroy 1985), un aspecto que todavía pasa desapercibido para muchos. Así, Cox 1991 pp. 31-32 sugiere:

If teachers concentrate on pupils' competence in written Standard English, pupils will gain sufficient knowledge of Standard English to be able to convert this into competence in Spoken Standard English when appropriate. 
La consecuencia esperable de la enseñanza de una variante oral del inglés estándar podría significar, como pone de manifiesto Carter 1999, p. 159, «to teach them to speak in formal written English», una consecuencia nada aconsejable, que empobrecería la competencia comunicativa de los estudiantes, al no promover la adaptación y adecuación al contexto situacional.

Además, es evidente que la definición de un posible estándar oral es todavía más imprecisa que en el caso de una variedad escrita, ya que la primera es más permeable al cambio y, por ello, menos susceptible de estandarización. Pero, en todo caso, la mayor parte de las descripciones de estándar (escrito u oral) aluden no a su estructura o rasgos definitorios, sino a sus potenciales hablantes. De hecho, según advierte Joseph 1987, p. 126:

Adherence to them [standard languages], identifies members of the upper social classes; but the standards themselves are largely defined by upper-class usage.

\section{LA TENDENCIA A LA ECUACIÓN (LENGUA ESTÁNDAR = LA LENGUA CORRECTA)}

Como consecuencia de las actitudes muy positivas hacia las particulares variedades estándares, que han cristalizado en definiciones poco científicas que equiparan «estándar» con «una forma de lengua [...que se emplea] corrientemente como el mejor medio de comunicación» (Dubois y otros 1973, p. 244), se asiste pronto a la afirmación de que es la única lengua correcta.

Ya en Elementary Lessons in English Grammar de Wyld 1909, se lee:

Our business is only with one main form of English, that form that is generally called Educated English, that is a sort of general average English which has a wide circulation among educated people, and is what is generally referred to by the rather vague name correct English, or better, Standard English [citado en Crowley 1989, p. 157; la cursiva es mía]

En la prensa, es muy común la aparición de fragmentos como el siguiente del Independent on Sunday, en que se afirmaba:

SE is grammatically correct English.... so that you can be understood clearly, so that you don't speak sloppily, you use tenses and prepositions properly, you don't say "He done it and you don't split infinitives...» He done it' is speaking English incorrectly. That's bad grammar. We think it important that our children speak correctly; [citado en Cameron 1995, p. 102.]

Lo sorprendente no es que tal actitud prescriptiva sea manifestada por la mayoría de profanos en la materia, sino que se constate en la plu- 
ma de algunos lingüistas, en los albores del siglo XXI. Así las cosas, es cierto que la mayoría rechaza contundentemente esta visión, muy inculcada en las escuelas, que desatiende que el resto de variedades lingüísticas no estándares tienen sus propias pautas de corrección, sus propias normas objetivas, pese a que carezcan de una codificación explícita, prescriptiva. En efecto, tal y como defiende Bartsch 1987, p. 269:

The standard is used as an outside measure of correction such that nonstandard speech seems to be incorrect speech, although it is only incorrect to the norms of the standard varieties, which are not its norms.

Méndez García de Paredes 2007 cree que la equiparación entre lengua estándar y lengua correcta «es totalmente inapropiada», y trasluce una actitud purista de aquellos para quienes la variedad codificada es «la lengua toda, [...] el único modelo de corrección e incluso [...] la única lengua posible».

Esta cuestión obliga a tratar la problemática relación entre el concepto de estándar y la noción de norma, que está presente en la mayor parte de las definiciones o descripciones del primero, y que debe tomarse como pieza clave en el camino en la investigación sobre una teoría de estandarización lingüística.

La norma [está] a medio camino entre el plano meramente gramatical y el del uso, el estándar de un modo particular (Pascual y Prieto de los Mozos 1998, p. 83).

Para Bernárdez 1999, p. 39:

Si hablamos la lengua normativa estamos hablando la lengua estándar, pero no siempre ni necesariamente a la inversa.

Según propone Demonte 2001, estándar sería una noción cercana a la norma social coseriana y, en el caso de la lengua española, ambos conceptos son inseparables «porque la LE no refleja ya un patrón de dominio de un dialecto sobre otro, sino un patrón de complementariedad y coherencia». También para Gallardo 1978 y Solà 2000, el sentido de norma que propuso el maestro rumano, definido como el primer grado de abstracción entre sistema y habla, forjado en el seno de la comunidad histórica, es el más próximo al concepto de estándar. Recuérdense las propias palabras de Coseriu 1962, pp. 90-98:

Aclaramos, además, que no se trata de la norma en el sentido corriente, establecida o impuesta según criterios de corrección y de valoración subjetiva de lo expresado, sino de la norma objetivamente comprobable en una lengua, la norma que seguimos necesariamente por ser miembros de una comunidad lingüística, y no aquella según la cual se reconoce que «hablamos bien» o de manera ejemplar, en la misma comunidad. Al comprobar la nor- 
ma a la que nos referimos, se comprueba cómo se dice, y no se indica cómo se debe decir $[\ldots]$ Cabe, sin embargo, señalar que muchas veces no coinciden, dado que la «norma normal» se adelanta a la «norma correcta», es siempre anterior a su propia codificación. [...] La norma es, en efecto, un sistema de realizaciones obligadas, de imposiciones sociales y culturales, y varía según la comunidad.

Coseriu distingue entre «norma normal», objetiva, implícita en los hablantes de cada comunidad, que sigue sus propias normas de prestigio, y que ve en determinados escritores, políticos, periodistas, etc. sus mejores representantes («normativización informal», siguiendo a Stewart 1968) y «norma correcta», entendida ésta como la norma ideal, explícita, formal y prescriptiva, recogida en tratados gramaticales y diccionarios (la «normativización formal», Stewart 1968). Según esto, el estándar sería esa «norma normal» o «norma social», de la que se percataron muy bien los lingüistas de Praga.

Sin embargo, si se defiende que la codificación y prescripción de la norma o, mejor dicho, normas del estándar, es un requisito imprescindible, aunque no suficiente, para la estandarización de una variedad y, por otro lado, se argumenta que el estándar es equivalente a la norma social coseriana, no necesariamente prescrita y codificada, la contradicción es evidente. Aún así, creemos que ambas condiciones pueden reconciliarse y compatibilizarse.

En primer lugar, conviene remitirse a los llamados «estándar absoluto» y «estándar relativo». El estándar en su grado más absoluto coincidiría con esa norma ideal, abstracta, de la que son más conscientes los hablantes por su codificación explícita, lo cual le otorga una mayor fuerza normativa: el «prescriptive standard» (Bartsch 1985, 1987) o «norma asintótica» (Rona 1973).Junto a esta norma a la que se tiende en mayor o menor medida, pero que es irrealizable por ser un «constructo mental» (Borrego 2001), se halla la norma social coseriana o, mejor dicho, las «normas sociolingüísticas», (Rona 1973), equivalentes a la norma de prestigio, un concepto próximo al «empirical standard» (Joseph 1987) o «relative standard» (Bartsch 1985, 1987). Se trata de una norma naturalmente descriptiva y objetiva, que tiene su correlato en los usos de los hablantes que se consideran modélicos, quienes se ajustan en mayor o menor medida a esa idealización ${ }^{9}$.

Aquellos lingüistas que resaltan que debería hablarse de una ideología de la estandarización, dado que se promueve el empleo de una variedad uniforme e idealizada, que no se corresponde con la natural

\footnotetext{
${ }^{9}$ A este respecto, resulta interesante la propuesta de Wolfram y Schilling-Estes 1998, pp. 10-11, quienes diferencian, dentro del estándar americano, entre un nivel formal y otro informal. El primero parece equipararse al Prescriptive Standard English (escrito y codificado), mientras que el segundo parece más en la órbita del llamado empírico.
} 
heterogeneidad dialectal, parecen hacerse eco de un «estándar absoluto o prescriptivo», al que tienden los hablantes, pero que sólo se alcanza cuando dicha variedad adquiere el estatus de lengua clásica y, por lo tanto, se insiste en que nadie habla propiamente el estándar. Quienes, por el contrario, hacen más hincapié en su vertiente sociohistórica y en la flexibilidad del estándar parecen remitir al «relativo o empírico», este sí realizable y alcanzable por los hablantes. Este concepto sirve para conjugar la variabilidad a la que aluden muchos lingüistas, que se concreta en sus realizaciones tanto escritas como orales. En este tipo piensan quienes acercan el estándar al concepto coseriano de norma social, que no tiene por qué estar codificada, en el que tendrían cabida los llamados «estándares nacionales o regionales», de los que hablan Kachru 1985, Österreicher 2004, 2006.

\section{El TRINOMIO LENGUA ESTÁNDAR - LENGUA COMÚN NO MARCADA - LINGUA FRANCA}

Tal y como se anticipó anteriormente, algunos lingüistas se refieren al estándar como un complejo que «tiene variantes, que van desde la lengua coloquial, o lengua familiar, hasta la académica o solemne ${ }^{10}{ }_{\gg}$ (Alcaraz Varó y Martínez Linares 1997, p. 323). En este sentido, tienden a argüir que el estándar es la lengua común o general que, para Cerdà 1986, p. 107,

sirve de vehículo comunicativo para cubrir todas las necesidades sociales e individuales de los miembros de una comunidad.

No obstante, cuesta creer que el hecho de que el estándar se emplee fundamentalmente en la modalidad escrita, en el intercambio científico de ideas, en la escuela, en la administración, en los medios de comunicación, etc. sea, a su vez, el vehículo adecuado para la conversación cotidiana y espontánea. No parece, pues, muy pertinente identificarla con la lengua empleada comúnmente, salvo que se pretenda «hablar como un libro».

A la identificación del estándar con la lengua común debió de contribuir sobremanera la adopción de los términos Gemeinsprache (Paul 1880), common language (Jespersen 1925) y langue commune (Vendryes 1921), que son equivalentes al estándar en términos absolutos, como ideal de calidad, y no la lengua empleada comúnmente (más cercana, en lo esencial, a la «norma» de Coseriu). Hermann Paul 1880, p. 404 exponía:

\footnotetext{
${ }^{10}$ Cuando se afirma que en el estándar hablado existen estilos formales e informales, a nuestro entender, debe tenerse presente que no se llega nunca al nivel de coloquialidad de los dialectos o variedades que se emplean comúnmente en la conversación ordinaria, no cuidada.
} 
Die Gemeinsprache ist natürlich erst recht eine Abstraktion. Sie ist nicht ein Komplex von realen Tatsachen, realen Kräften, sondern nichts als eine ideale Norm, die angibt, wie gesprochen werden soll. Sie verhält sich zu der wirklichen Sprechtätigkeit etwa wie ein Gesetzbuch zu der Gesamheit des Rechtslebens in dem Gebiete, für welches das Rechtsbuch gilt, oder wie ein Glaubenbekenntnis, ein dogmatisches Lehrbuch zu der Gesamheit der religiösen Anschauungen und Empfindungen ${ }^{11}$.

De hecho, el inglés estándar es, como advierte Crystal 1995, p. 110, la variedad de una minoría a la que se asocia mayor prestigio:

Altough SE [Standard English] is widely understood, it is not widely produced. Only a minority of people within a country (e.g. radio newscasters) actually use it when they talk.

El carácter supradialectal del estándar lleva, en muchas ocasiones, a tildarlo de lingua franca y a su identificación con la lengua empleada comúnmente. Este hecho se explica porque uno de los principios de configuración de un estándar es la selección de una base común ${ }^{12}$ a todas las variedades de una lengua, que abandona y neutraliza determinados rasgos formales en favor de una mayor eficacia comunicativa, como sucede con el estándar español. Sin embargo, la construcción de un estándar puede no basarse en un principio de coherencia o en un «sistema nuclear» (Mújica 2004), sino en un dialecto social o geográfico. Pero, la cuestión radica en que si bien la lengua estándar puede funcionar como «un instrumento de entendimiento común» (Seco 1972, p. 251), ello no implica que sea la lengua empleada generalmente en la conversación cotidiana.

En cambio, una koiné, que se forma con un núcleo común a las diferentes variedades, con el propósito de facilitar la comunicación transregional, en tanto que se utiliza para la expresión ordinaria de todos los hablantes, sí funciona como lengua común. Gallardo 1978, p. 98 precisa que el estándar sería una especie de koiné, pero muy codificado. De hecho, la variedad estándar más bien funciona como lingua franca en los usos cultos propios de los ámbitos formales, frecuentados por las clases más instruidas. En efecto, no se materializa en el habla cotidiana y espontánea de ninguno de sus hablantes.

¿No será que la supuesta identidad del estándar y el español usado normalmente es más un deseo que una realidad? (Pascual y Prieto de los Mozos 1998, p. 77).

11 «La lengua estándar es, naturalmente, una abstracción. No es un complejo de hechos reales, de fuerzas reales, sino nada más que una norma ideal, que indica cómo se debe hablar. En relación con la actividad lingüística real, se comporta como un código legal respecto de la vida jurídica de una región para la cual es válido, o como una profesión de fe, un catecismo dogmático respecto de las concepciones y las emociones religiosas.» [mi traducción].

12 «El criterio básico del estándar: lo que es común en mayor grado.» (Bernárdez 1999, p. 45). 
$\mathrm{Al}$ respecto, Moreno Cabrera 2008, p. 52 matiza que la koineización es un proceso natural en el que diversas variedades entran en contacto, se acomodan y construyen «un sistema adaptativo complejo [...que va] evolucionando para adaptarse a las circunstancias comunicativas». Por el contrario, como se ha puesto de relieve, en la configuración de un estándar el componente artificial es muy preponderante y, a diferencia de la koiné, surge en una situación de superposición, muy impulsada ideológicamente.

A esta consideración cabe añadir el hecho de que una koiné no se erige en modelo idiomático de una comunidad, mientras que el estándar se convierte en «el punto de referencia central, a partir del cual se caracterizan otras modalidades de uso de la lengua»(Carbonero 2003b), «el parámetro .. calificador del estatus de los fenómenos lingüísticos», como apunta Österreicher 2006, p. 3080.

En consecuencia, dado el valor simbólico que adquiere una variedad estándar, codificada explícitamente, y su asociación con la elite social, política o económica, parece un tanto aventurado caracterizarla como «una lengua neutra, no marcada» (Mújica 2004) o, como sugiere Moreno Fernández 1998, p. 336,

[...] aquella que está desprovista de cualquier marca diacrónica, diatópica, diafásica, diastrática-alejada de una norma general.

Pese a que el estándar funciona como marco de referencia frente al que se concreta el espacio variacional (Carbonero 2003b, Méndez García de Paredes 2007), tal y como pone de relieve Demonte 2003, una variedad que se realiza "particularmente [...] en situaciones formales y, desde luego, en la modalidad escrita» no puede considerarse no marcada. Además, es necesario matizar que «es imposible que alguien pueda hablar sin marca alguna». Así pues, parece más plausible la opción de considerar la noción de estándar cercana a la de variedad diastrática y diafásica, connotada positivamente, propia del ámbito de la distancia comunicativa (Österreicher 2004, 2006).

Es indiscutible el carácter idealizado del estándar, un hecho en el que coincide el grueso de la bibliografía especializada (Haugen 1966; Bartsch 1985, 1987; Zamora Salamanca 1985; Joseph 1987; Moreno Fernández 1998; Bernárdez 1999; Martín Zorraquino 2001; Demonte 2003; Carbonero 2003a, 2003b; Moreno Cabrera 2008, etc.). No existe ningún hablante que condense todos los rasgos en un estándar, ni que, inversamente, pueda desposeerse de todas las características de su propio idiolecto. No en balde, los lingüistas del Círculo de Praga definieron al estándar en términos de propiedades, funciones y actitudes «continuous, sliding-scale features rather than discrete, yes-no features» (Garvin 1959 , p. 522). 
Así las cosas, la abstracción que subyace al estándar no es, a nuestro juicio, equivalente al concepto teórico saussureano de langue (Alvar 1990). Citando a Demonte 2003:

No se define como modelo de competencia sino como modelo de comprensión, adecuación y propiedad, es decir, como modelo que tiene que ver con el uso y no con el conocimiento de una lengua.

En la inadecuación de tal identificación insisten también Pascual y Prieto de los Mozos 1998, quienes advierten de la existencia de un sistema, una langue propia para las variedades no estándares. Asimismo, Gallardo 1978, p. 86 insiste en la presencia de elementos de la parole en el estándar que, a diferencia de la langue, «está delimitado por la cultura».

\section{ConClusión}

Este trabajo ha puesto de relieve cómo las diferentes definiciones de «estándar» reflejan la motivación social, política y económica que explica su surgimiento. Efectivamente, el proceso de estandarización es, indudablemente, un ejemplo de cambio lingüístico deliberado, cuyo resultado no coincide con el idiolecto de ningún hablante, por lo que muchos lingüistas han aludido a él como un proceso contrario a la evolución natural de las lenguas

Así las cosas, este hecho no impide que este particular tipo de elaboración lingüística sea una necesidad para la cohesión y la movilidad social, una consecuencia directa de la democratización de la vida pública y una manifestación más del ser histórico de una lengua. No puede menospreciarse la siguiente afirmación de Borrego Nieto 2001:

La ideología de la estandarización es indispensable para la moderna difusión masiva de la información, a la vez que se apoya en ella para consolidarse.

Ante todo, este artículo ha puesto de relieve que cada variedad lingüística tiene sus propias normas de corrección, sus ejemplaridades idiomáticas, su valor y su función en el complejo entramado de la comunicación humana, pero la base del estándar es la norma culta, «la variedad formal», en palabras de Wagner 1983.

Si se desea llevar a cabo una mejor aproximación y perfilar una definición para «estándar», «una realidad sociolingüística, que puede y debe convertirse en el punto de referencia de todos los estudios normativos» (Gutiérrez Ordóñez 2001), no puede olvidarse la existencia de «las otras normas». Estas normas sociolingüísticas, pragmáticas, discursivas, culturales son tan importantes como las propiamente gramaticales y en ellas también «habría que buscar las huellas de los estánda- 
res» (Prieto de los Mozos 2001), al contrario de lo que ha sido la tónica general en las gramáticas y manuales de reflexión normativa.

\section{REFERENCIAS BIBLIOGRÁFICAS}

Alba, O. (2001): «El español estándar desde la perspectiva dominicana», en II Congreso Internacional de la Lengua Española, Valladolid, (Disponible en: $<$ http://www.congresosdelalengua.es/valladolid/ponencias/unidad_diversidaddel_espanol/1_la_norma_hispanica/alba_o.htm>.

Alcaraz Varó, E. y Martínez Linares, M. A. (1997): Diccionario de lingüistica moderna, Barcelona, Ariel.

Alvar, M. (1990): «La lengua, los dialectos y la cuestión de prestigio», en Moreno Fernández, F. (recop.), Estudios sobre variación lingüistica, Alcalá de Henares, Universidad de Alcalá de Henares, pp. 13-26.

APPEL, R. y MuYsKen, P. (1987): Language contact and bilinguism, Londres, Arnold (trad. esp.: Bilingüismo y contacto de lenguas, Barcelona, Ariel, 1996).

BakHTin, M. (1981), The dialogic imagination: Four essays by M. M. Bakhtin, M. Holquist (ed.), Austin, University of Texas Press.

BARTSCH, R. (1985): "The influence of language standardization on linguistic norms», Studia Linguistica 1, 39, pp. 23-50.

-, (1987): Norms of language. Theoretical and practical aspects, Londres/Nueva York, Longman.

BERnÁRdeZ, E. (1999): ¿Qué son las lenguas?, Madrid, Alianza Editorial.

Borrego Nieto, J. (2001): «El concepto de norma regional y su aplicación a las hablas castellano-aragonesas», en II Congreso Internacional de la Lengua Espanola, Valladolid, <http://congresosdelalengua.es/valladolid/ponencias/ unidad_diversidad_del_espanol/1_la_norma_hispanica/borrego_j.htm>.

CAMERon, D. (1995): Verbal hygiene, Londres/Nueva York, Routledge.

Carbonero, P. (2003a): «Norma estándar y actitud sociolingüística», Estudios de sociolingüistica andaluza, Sevilla, Secretariado de Publicaciones de la Universidad de Sevilla, pp. 21-29.

—, (2003b): «Problemas de la nivelación lingüística del español actual», Estudios de sociolingüistica andaluza, Sevilla, Secretariado de Publicaciones de la universidad de Sevilla, pp. 31-38.

Cardona, G. R. (1978): Dizionario di linguistica (trad. esp. Diccionario de lingüistica, Barcelona, Ariel Lingüística, 1991).

CARTER, R. (1999): «Standard grammars, spoken grammars: Some educational implications», en Bex, T. y Watts, R. J., Standard English: The widening debate, Londres, Routledge, pp. 149-166.

Cerdà Massó, R. (coord.) (1986): Diccionario de lingüistica, Madrid, Anaya.

Chesire, J. (1999): «Spoken Standard English» en Bex, T. y Watts, R. J., Standard English: The widening debate, Londres, Routledge, pp. 129-148.

COOPER, R. L. (1989): Language planning and social change, (trad. esp. La planificación lingüistica y el cambio social, Cambridge, Cambridge University Press, 1997). 
Coseriu, E. (1962): «Sistema, norma y habla», Teoría del lenguaje y lingüística general. Cinco estudios, Madrid, Gredos, pp. 11-113, 1989.

Cox, C. B. (1991): Cox on Cox: An English curriculum for the 1990s, Londres, Hodder and Stoughton

Crowley, T. (1989): Standard English and the politics of language, Hampshire/Nueva York, Palgrave Macmillan, 2003.

CRYstal, D. (1995): The Cambridge Encyclopedia of the English language, Cambridge, Cambridge University Press.

DAviEs, A. (1997): "Real language norms: description, prescription and their critics. A case for Applied Linguistics», Viena English Working Papers 6, 2, pp. 4-18.

Demonte BARReto, V. (2003): «Lengua estándar, norma y normas en la difusión actual de la lengua española», Circunstancia 1, pp. 1696-1277, disponible en $<$ http://.www.ortegaygasset.edu/circunstancia/numero1/art4_imp.htm>.

Department of Education AND SCIEnce (1988): Report of the Committee of Inquiry into the Teaching of English Language (Kingman Report), Londres, HMSO.

Deumert, A. y Vandenbussche, W. (2004): «Standard languages. Taxonomies and histories», en Deumert, A. y Vandenbussche, W. (eds.), Germanic standardisation. Past to present, Ámsterdam/Philadelphia, John Benjamins, pp. 1-14.

DITTMAR ET AL. (eds.) (1988): The sociolinguistics of urban vernaculars, Berlín/Nueva York, Walter de Gruyter.

Downes, W. (1984): Language and society, Cambridge/Nueva York/Melbourne, Cambridge University Press, 1998.

Dubois, L. ET AL. (1973): Dictionnaire de Linguistique, (trad. esp. Diccionario de Lingüistica, Madrid, Alianza Editorial, 1979).

FAirclough, M. (2001): «La adquisición de un segundo dialecto: el español estándar en una situación de contacto», disponible en <http://www.ub.es/ice/ portaling/seminari/seminari_pdf/52fairclough.pdf $>$.

FERNÁNDEZ RAMírez, S. (1985): Gramática española, Madrid, Arco/Libros

GARVIN, P. L. (1959): «The standard language problem», en Hymes, D. (ed.), Language in Culture and Society, Nueva York, Harper and Row, pp. 521-526.

Gallardo, A. (1978): «Hacia una teoría del idioma estándar», Revista de Lingüistica Teórica y Aplicada 16, pp. 85-119.

Garrido Medina, J. (1996): Estilo y texto en la lengua, Madrid, Gredos.

GrACE, G. W. (1990): «Is contemporary linguistic theory an instrument of cultural imperialism», Ethnolinguistic Notes 3, 41, pp. 1-19.

GutiÉRREZ ORDÓÑEZ (2001): «Perfiles y dimensiones del concepto de norma, las otras normas», en II Congreso Internacional de la Lengua Española, Valladolid, disponible en <http://www.congresosdelalengua.es/valladolid/ponencias/ unidad_diversidad_del_espanol_/1_la_norma_hispanica/gutierrez_s.htm>.

HAAs, W. (1982): «On the normative character of language», en Haas, W. (ed.), Standard languages: Spoken and Written, Manchester, Manchester University Press, pp. 1-36.

Hají OMAR, A. (1991): «Standard languages: Its emergence and choice», en Tickoo, M. L. (ed.), Languages and standards. Issues, Attitudes, Case Studies, Singapur, SEAMEO Regional Language Centre, pp. 1-10. 
Haugen, E. (1966): Language conflict and language planning: The case of Modern Norwegian, Cambridge (Mass.), Cambridge University Press.

Hernández Alonso, C. (2001): «La norma y las normas. El español estándar» [Documento en línea], en II Congreso Internacional de la Lengua Española, Valladolid, disponible en <http://www.congresosdelalengua.es/valladolid/ ponencias/unidad_diversidad_del_espanol/1_la_norma_hispanica/hernandez_c.htm\#n9>).

—, (1968): «Language planning in Modern Norway», en Fishman, J. A. (ed.), Readings in the Sociology of Language, La Haya/París, Mouton \&Co, pp. 673$687,1970$.

Honey, J. (1997): Language is power: The story of Standard English and its enemies, Londres, Faber.

Hudson, R. A. (1980): Sociolinguistics, Cambridge, Cambridge University Press.

JESPERSEN, O. (1925): Mankind, Nation and Individual from a linguistic point of view, Londres, George Allen \& Unwin Ltd, 1946.

JOSEPH, J. E. (1987): Eloquence and power: the rise of language standards and standard languages, Londres, Frances Pinter.

—, (1991): «Levels of consciousness in the knowledge of language», en Tickoo, M. L. (ed.), Languages and standards: Issues, Attitudes, Case Studies, Singapur, SEAMEO Regional Language Centre, pp. 11-22.

KACHRU, B. B. (1985): «Standards, codification and Sociolinguistic realism: The English language in the outer circle», en Quirk, R. y Widdowson, H. (eds.), English in the world: Teaching and learning the language and literatures (Papers of an International Conference held to celebrate the $50^{\text {th }}$ Aniversary of the British Council), Cambridge, Cambridge University Press, pp. 11-30.

Kratochvil, P. (1968): The Chinese language today: Features of an emerging standard, Londres, Hutchison.

LARA, L. F. (1976): El concepto de norma lingüística, México, El Colegio de México. LEITH, D. (1983): A social history of English, Londres, Routledge \& Kegan Paul.

LÉvi-Strauss, C. (1952): Raza e historia. Raza y cultura, Barcelona, Edicions 62, 1985.

LeWANDOWSKI, T. (1982): Diccionario de Lingüistica, Madrid, Cátedra.

MARTín ZorraQuino, M. A. (2001): «El neoespañol y los principios que fundamentan la lengua estándar o consagrada» [Documento en línea], en $I I$ Congreso Internacional de la Lengua Española, Valladolid, disponible en $<$ http://www.congresosdelalengua.es/valladolid/ponenecias/unidad_diversidad_del_espanol_/1_la_norma_>

MCWorther, J. (2001): The power of Babel. A natural history of language, Nueva York, Henry Hock and Company.

MÉndez Garcia de Paredes, E. (2007): «Modelos idiomáticos, codificación de usos y prescriptivismo», en Coloquio Internacional In Memoriam Manuel Alvar. Variación lingüistica y contacto de lenguas en el mundo hispánico (en prensa).

—, (2008): «Modelos idiomáticos y prescriptivismo. El caso del andaluz», en Moreno Sandoval, A. (ed.), Actas de VIII Congreso Internacional de Lingüistica General. El valor de la diversidad [metal]lingüistica, Madrid, Universidad Autónoma de Madrid, disponible en <http://elvira.lllf.uam.es/clg8/actas/ index.html>. 
Milroy, J. y Milroy, L. (1985): Authority in language. Investigating language prescription \& standardisation, Londres, Routledge, 1991.

Moreno Cabrera, J. C. (2008): El nacionalismo lingüístico. Una ideología destructiva, Madrid, Ediciones Península.

Moreno Fernández, F. (1998): «La planificación lingüística», en Moreno Fernández, J. (ed.), Principios de sociolingüistica y sociología del lenguaje, Barcelona, Ariel Lingüística, pp. 331-343.

MúJICA, N. (2004): «Norma lingüística y variaciones: algunos casos problemáticos en el español de Argentina», [Documento en línea], en III Congreso Internacional de la Lengua Española, Rosario, disponible en $<\mathrm{http}$ //www.congresosdelalengua.es/rosario/ponencias/aspectos/mujica/n.htm>

Murray, J. A. H. ET AL. (1933): Oxford English Dictionary, Oxford, Clarendon Press, 1989.

MyHiLl, J. (2004): «Why has Black English not been standardized? A cross-cultural dialogue on prescriptivism», Language Sciences 26, pp. 27-56.

O’ Grady, W.; Dobrovolsky, M. y Aronoff, M. (eds.) (1997): Contemporary linguistics. An introduction, Nueva York, St. Martin Press.

ÖSTERREICHER, W. (2004): «El problema de los territorios americanos», [Documento en línea], en III Congreso Internacional de la Lengua Española, Rosario, <http://www.congresosdelalengua.es/rosario/ponencias/aspectos/ oesterreicher_w.htm>).

—, (2006): «El pluricentrismo del español», en Actas del VI Congreso Internacional de Historia de la Lengua Española, Vol. III, Madrid, Arco/Libros, pp. 30793087.

Pascual Rodríguez, J. A. y Prieto de los Mozos, E. (1998): «Sobre el estándar y la norma», en Kent, C. y De la Calle, M. D. (eds.), Visiones salmantinas, Salamanca, Universidad de Salamanca/Ohio Wesleyan University, pp. 63-95.

Paul, H (1880): Prinzipien der Sprachgeschichte, Tubinga, Max Niemeyer Verlag, 1975.

PenNy, R. (2000): Variación y cambio en español, Madrid, Gredos.

PRIETO DE LOS Mozos, E. (1999): «Sobre las gramáticas normativas y las gramáticas no normativas», en Homenatge a J. Tusón, Barcelona, Empúries, pp. 245258.

—, (2001): «Variación, norma y aprendizaje lingüistico», [Documento en línea], en II Congreso Internacional de la Lengua Española, Valladolid, disponible en $<$ http://www.congresosdelalengua.es/valladolid/ponenecias/unidad_diversidad_del_espanol_/1_la_norma_hispanica/prieto_e.htm $>$.

QUIRK, R. ET AL. (1985): A contemporary grammar of the English Language, Londres, Longman.

Rojo, G. (1986): El lenguaje, las lenguas y la lingüistica, Santiago de Compostela, Universidad de Santiago de Compostela.

Romaine, S. (1988): «Language in society: An introduction to sociolinguistics», Oxford, Oxford University Press (trad. esp. El lenguaje en la sociedad: Una Introducción a la Sociolingüistica, Barcelona, Ariel, 1996).

RonA, J. P. (1973): «Normas locales, regionales, nacionales y universales en la América española», Nueva Revista de Filología Hispánica 22, pp. 310-321. 
Seco, M. (1972): Gramática esencial del español, Madrid, Espasa-Calpe, 1991.

Silva Corvalán, C. (2001): Sociolingüistica y pragmática del español, Washington DC, Georgetown University Press.

SLedD, J. (1969): «Bidialectalism. The linguistics of white supremacy», English Journal 58, pp. 1307-1315.

SoLÀ, J. (2000): La terminologia lingüistica en l'ensenyament secundari. Propostes pràctiques, Barcelona, Fundació Caixa de Sabadell.

SPEICHER, B. L. y BiELANSKI, J. R. (2000): «Critical thoughts on teaching Standard English», Curriculum Inquiry 30, 2, pp. 147-169.

STEWART, W. (1968): «A sociolinguistic tipology for describing national multilingualism», en Fishman, J. A. (ed.), Readings in the Sociology of Language, La Haya, Mouton, pp. 531-545.

Steger, H. (1998): «Sprachgeschichte als Geschichte der Textsorten, Kommunikationsbereichten und Semantiktypen», en Werner, B., Reichmann, O. y Sonderregger, S. (eds.), Sprachgeschichte. Ein Handbuch zur Geschichte der deutschen Sprache und ihrer Erforschung, Vol. I, Berlín/Nueva York, Walter De Gruyter, pp. 288-300.

Strevens, P. (1983): «What is Standard English?», en Smith, L. E. (ed.), Readings in English as an International Language, Oxford, Pergamon Press. pp. 87-94.

SubaČIUS, G. (2004): «Review of Germanic standardisations. Past to present», Archivum Lithuanicum 6, pp. 351-358.

TAULI, V. (1968): Introduction to a theory of language planning, Uppsala, Acta Universitatis Upsaliensis, Studia Philologiae Scandinavicae.

Trudgill, P. (1999): «Standard English: what it isn't», en Bex, T. y Watts, R. J. (eds.), Standard English: The widening debate, Londres, Routledge, pp. 117128.

VAN MAARLE, J. (1997): «Dialect versus standard language: nature versus culture», en Chesire, J. y Stein, D. (eds.), Taming the vernacular: From dialect to written standard language, Londres/Nueva York, Longman, pp. 13-34.

Vendryes, J. (1921): Le language: Introduction linguistique a l'histoire, París, Albin Michel.

Voloshinov, V. (1929): Marxism and the philosophy of language, Nueva York, Seminar Press, 1973.

WAGNER, C. (1983): «La lengua formal, lengua ejemplar», Revista de Lingüística Aplicada 21, pp. 129-136.

Wolfram, W. y Schilling-Estes, N. (1998): American English: Dialects and variation, Malden/Massachussets/Oxford, Blackwell.

WyLD, H. C. (1909): Elementary lessons in English grammar, Oxford, Clarendon Press.

—, (1934): The Best English: A claim for the superiority of Received Standard English, Oxford, Clarendon.

Zamora Salamanca, F. J. (1985): «Sobre el concepto de norma lingüística», Anuario de Lingüistica Hispánica 1, Universidad de Valladolid, Secretariado de publicaciones de la UVA, pp. 227-249. 